\title{
Established Mass Customization in Highly Customized Cabins of Passenger Transport Systems
}

\author{
Felix Schulz ${ }^{1}$, Lars Wolter ${ }^{2}$, Turgay Coruh ${ }^{3}$, Haygazun Hayka ${ }^{1}$, and Rainer Stark ${ }^{1,2}$ \\ ${ }^{1}$ Fraunhofer IPK Berlin, Germany \\ ${ }^{2}$ Technical University Berlin, Germany \\ ${ }^{3}$ Airbus Operations GmbH, Germany \\ felix.schulz@ipk.fraunhofer.de
}

\begin{abstract}
Mass customization is an approach that provides methodologies for the development of customized products while still profiting from the benefits of mass production such as economies of scale. Today, mass customization approaches have found their way into different industries such as clothes and furniture but also, to some extent and named differently, into more complex products like cars. Products like busses, trains, or aircrafts are not particularly known for applying similar approaches. They deal with complex systems that are highly regulated by legal requirements and thus seem to be restricted with respect to their possible degree of customization. This paper presents results from a study that was conducted in order to analyze applied practices in these industries. One of the goals of the study was to detect limitations of today's mass customization approaches that prevent an easy applicability. Configuration and customization approaches in the airplane cabin design, in the bus and in the train industry were investigated and compared. Finally, shortcomings in the applied practices and in the supporting solutions were identified and suggestions for improvements were formulated.
\end{abstract}

Keywords: Mass Customization, Configuration.

\section{Introduction}

This paper illustrates that the branches bus, train, and aircraft manufacturing industry are facing challenges that are comparable to problems addressed by mass customization approaches. Furthermore, it will be explained that common approaches for the investigated branches are not sufficient, due to multiple reasons. This information shall be used in order to derive suggestions for improvement of the current approaches. This enables an application of methods for more customizable complex products.

The outcome of this paper can be used for enhancing the use of mass customization strategies for industries that offer products with a higher degree of customizability.

\section{State of the Art in Mass Customization}

In order to discuss needs regarding configuration management in the investigated field it is necessary to define a uniform terminology used throughout this paper. 
This terminology is coming from general language within the investigated branches and was extended and consolidated by the authors for better understanding. First of all, configurable and highly customizable products - like buses and trains- usually only exist as virtual products during the offering of the product. By contrast, products that are offered in a pick-to-order business model do already physically exist during the ordering process. It is, therefore, necessary to differentiate between a product, a product line and a physically existing product instance. The product instance is the configured product to meet the individual needs by customization or using a set of configuration options. Further differentiation is necessary between configuring within a closed catalogue of options, common in the automobile industry, and open configuration which allows the creation of more customer-specified options. For the latter case the customer defines requirements in so called Customer Special Requests (CSR) that describe a single feature or combination of feature which is not in any catalogue of the company.

The general approach for mass customization is used for products that are configurable without having CSRs. This is true for computers, shoes, cars and many other products. It is not the case for products like busses, trains, airplanes and ships. Therefore, the general methods that handle mass customization are mainly applicable to the first kind of products. The increased need of producing large numbers of CSR driven products may make it desirable to use mass customization methods during their development.

There are no consolidated typical characteristics of mass customization. Several sources mention different aspects or so called mass customization characteristics. Kumar deals with mass customization, its relation to business strategy and its demand within a company's supply chain and management [1]. According to that, mass customization is characterized or usually associated with a customer co-design process, a finite solution space, and normally low production cost per unit.

Moser identifies the strategic considerations companies follow when pursuing the mass customization concept and differentiate the existing types of mass customization in his research [2]. In industry studies he detected that one characteristic is that in mass customization there exists intensive customer integration. According to Wikström [3], an application of product configuration systems is necessary to compose a mass customized product. The consumer decision-making process is often a complicated process. Bettman writes about this increased decision-making effort in his study [4]. Petruzellis addresses this problem in his studies about bundling of configurable product items [5]. A decision-making process is influenced by enormous number of criteria like decision-making strategy, individual's character, kind of presentation of alternatives and options. Therefore companies need to reduce choice complexity as demanded by Petruzzellis and Chatterjee [5], [6]. An employment of product modularity is usually seen in companies that use the mass customization approach, which is a basic principle to modularize product architectures. A broad product variant management is claimed to be necessary. Furthermore it is necessary to establish a central production and logistics planning as well as a management of mass and individual production. Mäkipää describes this with: "customization strategies often require a high technology production environment" [7]. A special 
management of flexible organization processes is required to achieve a flexible production environment. Also a process documentation and IT support is required

Mäkipää and Mertanen identify similar elements in their study about the application-level of mass customization approaches within the Finnish industry [7], [8]. These elements are a customer integration and relationship management, a high focus on product development, manufacturing resource planning and procurement, after sales, special management, and other factors like organizational commitment and creativity. The contents of these topics are more or less comparable to the contents of the studies mentioned before. All referenced sources offer a comparable view on mass customization. Moser and Mäkipää offer more comparable aspects. Kumar offers a more reduced view on mass customization. This is due to the facts that he does not analyze the business change management required for mass customization.

It shows that there is a shared understanding of mass customization among researchers although there is no consolidated definition.

The tool landscape from the engineering perspective consists of multiple IT tools [7], [8] and can be clustered as shown in Figure 1. The IT systems used for configuration management are mostly depending on the user's role. Product (line) development is used to execute product data management (PDM) systems. Enterprise resource planning (ERP) systems arise from the need of production and manufacturing and as they manage the enterprise's resources they are closely linked to sales. Therefore today's ERP systems offer increasingly more functionalities for product configuration and to synchronize orders with production. Customer relationship management (CRM) systems are just as ERP systems not in the focus of this investigation and are usually sales-oriented. It is fair to say that each IT-Tool has its right to exist, because it is able to deal with the special requirements existing in each domain and no system is able to substitute all other systems. Therefore an integration of all the systems is achieved by having some kind of PLM infrastructure which more or less connects the different IT systems as found out in an expert survey ("Delphi-Studie 2020") about future PLM systems [9]. Aside from the infrastructure various data systems like IMDS for hazardous or dangerous materials are used throughout the whole lifecycle. Stark summarizes aspects of virtual product development in automotive industry. He mentions for example, that the demand for IT tools and methods in automotive industry is larger than it is in software development [10]. Consequently, during the whole lifecycle, it will be necessary to constantly bridge between a variety of information databases and authoring systems to handle patchwork solutions. Müller et al conducted a study about deficits and potentials of today's collaborative product development [11]. It shows that there is a rising demand for transparency within different IT systems to increase information logistics and project management.

Most of the product configuration tasks during product planning and development are handled by PDM systems. Modern PDM Systems offer different tools for configuration management and are normally linked to CAD authoring systems where geometric parameters are processed. Although all the systems mentioned are 


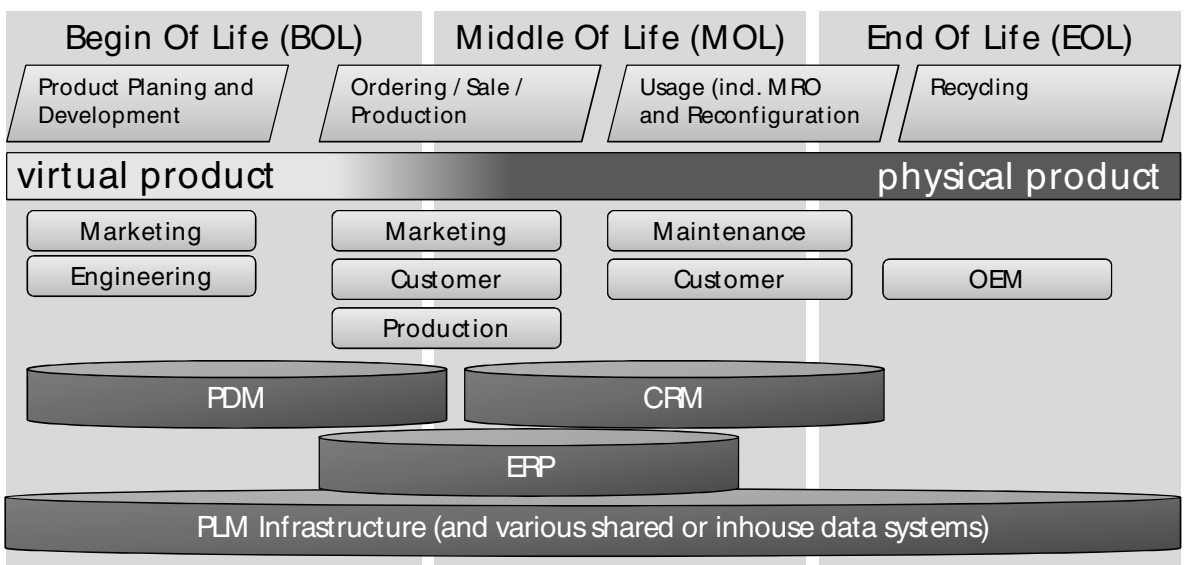

Fig. 1. Phases of product lifecycle, roles and range of influence of IT systems within lifecycle

specialized for engineering, there are different approaches to deal with product variation. One approach is to enrich the items with configuration meta data. The other approach is to establish logical links between items and possible variants. Further problem is that systems prefer to use their own standards for models and interfaces.

Using this flexible set of methods and tools the mass customization approach might be transferred to products using multiple CSRs during their ordering process. Different industries take slightly different approaches in realizing mass customization with a bit of fully individual customization wishes. To gain this knowledge, different industries have been analyzed during this study regarding their customization approaches focusing on the interior design.

\section{Research Approach and Data Ascertainment}

The study is carried out in three main phases. The overall approach is inspired by the first phases of the Six Sigma approach for problem solving. It is named DMAIC [1] as it consists of the phases to define the problem, measure the relevant measurement parameters, analyze the results of the measurement, improve a solution, and control the success of the improved solution. Since the purpose of this study does not include the improvement of a specific solution, the main phases considered are the definition and the measurement phase. An outlook on the improvement phase is given as the result of the study is used to give recommendations and improvement possibilities. Figure 2 shows the structure of the study.

The use of the Six Sigma DMAIC approach is on purpose limited to the concept and the use of the main generic phases. Six Sigma analysis tools are not used in this study. The initial definition phase investigates customization practice and the description of terms and solutions from a research point of view. Additional prescriptive studies where used to gather information about the industries using contacts of the Fraunhofer IPK. 


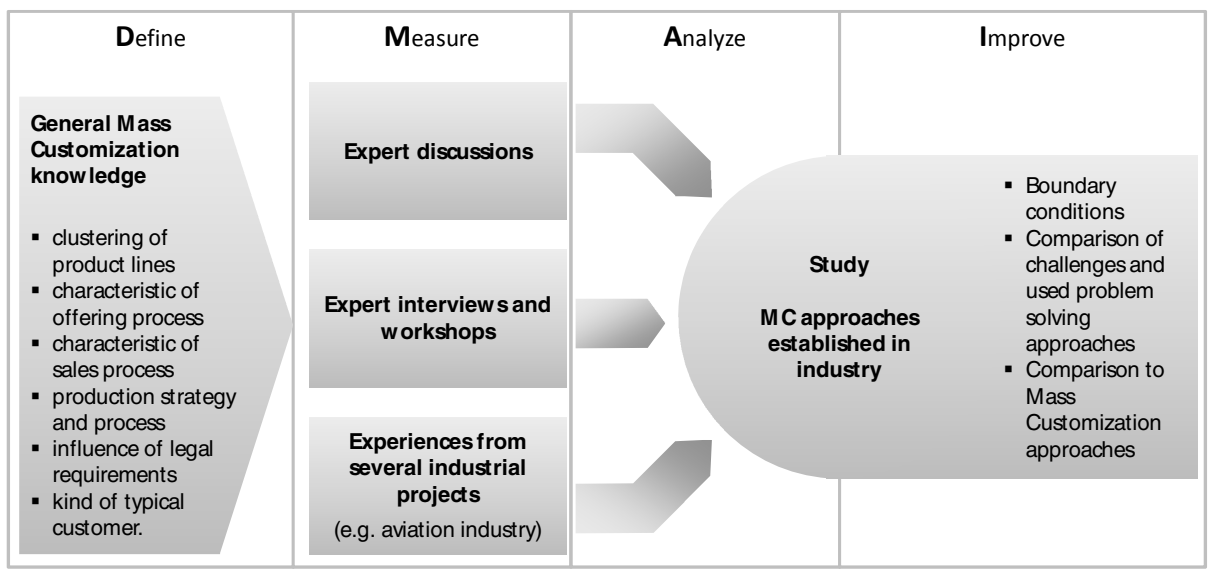

Fig. 2. The conduction of the study about mass customization (MC) approaches established in industry inspired by the DMAIC Approach

During the measurement phase multiple descriptive studies where performed and analyzed in an iterative manner. These studies included a small pre survey, telephone interviews and in depth workshops with experts from different companies of the bus and train industries. The workshops deepened the results from some of the telephone interviews. The interviewed experts were employees from different companies from automotive, train, and aircraft industry working as product managers, sales managers, development engineers, or IT managers. Three major results have been derived from this preliminary analysis: knowledge about the mass customization approaches established in the bus and train industries, the problems these industries are facing, and possible common solution approaches to solve these problems.

In addition, two expert discussions in 2011 and 2012 were set up during execution of the study and are discussed in Detail in chapter 5.

\section{Investigated Industries - General Overview}

Since the objective of this paper is to compare and to identify the applied practices of bus and train industries with respect to the cabin customization, there is the need to describe those two industries. Additional knowledge about automobile and aircraft industry extends this comparison. Knowledge about configuration approach and customizing practice within these branches is not cabin-specific as these branches were not core areas of the investigation.

The focus on cabin customization results from the complexity in this area. Customers purchasing a product commonly have very special wishes for the interior as it represents their company. Most of the cabins also introduce repeating elements and structures that allow a lot of automation during the configuration. This kind of flexibility between open configurable areas and closed configuration options together with the general understanding of the different cabin elements makes the cabin a perfect example and the study also applicable to other areas of product development. 
A difference exists concerning the type of the customer. Whereas typical buyers of coaches are often smaller private carrying companies, public transport busses and trains are almost exclusively sold in tendering procedures. In this case, the buyers are companies or governmental institutions. Airplanes are often sold either to airlines or to leasing companies. Caused by kind of typical customers, the characteristics of offering process are different, as well.

As this overview between the industries bus and train and the comparison to automotive and aircraft industry shows, different business models and different production philosophies are in use but the industries are still comparable, especially when looking at the cabin interior. The expected similarities between these industries are therefore validated and hints for detailed investigations are identified.

\subsection{Bus Industry}

The development of busses is following an engineer-to-order approach and differs in time from several weeks for coaches to several months for city busses. Customization of busses is standard. There are two different segments of busses, which should be differentiated. The first segment is "city busses". The second segment is "coaches" which are usually used for long distance travelling. From an engineering perspective, there are differences in terms of floor height and customization degree. City busses typically are ordered in tendering procedures and widely specified by customers such as regional operators (cities). As these operators often maintain busses in own workshops, they have broad requirements concerning maintenance, repair and overhaul (MRO), such as colors of specific cables, use of switch connector fuses and so on. Furthermore highly specified requirements concerning corporate design, ticket machine interfaces, seats, handles, stop-buttons, and so on are common. Design parameters for those elements include number, model, position constraints like seat pitch, colors, materials, and so on. These busses are typically ordered in higher take rates than long distance coaches. City busses for instance may be ordered in series of five to 20 (in few cases approx. 80) busses.

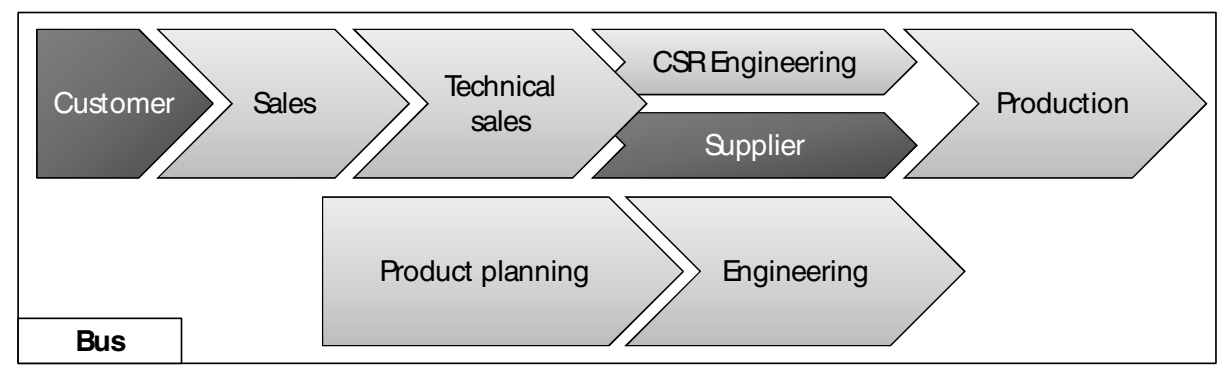

Fig. 3. Abstract bus development process. Ordering of busses happens in the upper horizontal lane. Non-CSR Engineering handles productline development and specific customer wishes. 
Long distance coaches do have less customization although offering higher degrees of comfort, advanced designs (in the sense of styling) and media features. One reason is the customer segment, which mainly consists of operators with smaller fleets. Coaches are typically ordered in single items; in few cases series of 5 same items are ordered.

The development process is standardized and well structured. An abstracted view is presented in figure 3. According to a configuration, the top level product structure and bill of material become defined and are filled with design solutions during the engineering process. The product structure is a flat, 3 levels deep in average, structure for every product line defining the modules that each bus consists of on the first level. Products are configured within this 150\%-structure to reach 100\%-structure. Design solutions from former projects are adapted to the requirements of the new project and considered like "standard components" in a serial development process. More challenging requirements (special customer requests, CSR) are treated separately with regular customer interaction and more engineering time in parallel to the serialized standard process which is also common to the automotive industry. Usually numbers of CSRs within coaches are in one-digit levels. City buses usually have hundreds of CSRs. Special customer requests that have been realized as design solutions become part of the standard module catalogue if promising for later projects.

The design of coaches and city busses is based on platforms and modules. Validation of module fit to customized configurations is necessary during the design process and performed each time particularly. The investigated cases provide a reference that there are different degrees of competence in companies to validate possible customized configurations already during the offering process. In one case, the validation was quick because extended design verification and validation against the standard catalogue is carried out more extensively while developing design solutions for specific customer request. This raises effort the first time but pays off in case of high take rates in later projects.

Systematization of IT tool chains is established and continuously evolved, because the development process and the parts and document management (product data management) is common for all projects.

\subsection{Train Industry}

The development of trains is driven by customer projects and tendering procedures. Train development follows the engineer-to-order (EtO) approach and development time can take up to several years. There are typically different train types that are built for different requirements and operations. The main aspect for the customization and development project processes are:

- Train type: high velocity train, intercity trains, region trains, and city trains

- Customer type: large customers, small operators

- Role in the supply chain: (contractor / tenderer, supplier)

- Dependencies of railroad network (different tracks and electrical power supply) 
- Performance parameters: speed, acceleration, transportation capacity

- Design parameters to be mentioned (min. curve radius, envelop curve, and track gauge)

High velocity trains, regionally operated trains as well as trams do have significant differences in terms of customer requirements and design. In general, the design of trains is driven by customer-driven functional and style requirements, national requirements, regional requirements (e.g. height of station platforms) and performance requirements.

Trains are produced in small series between 10 and 40 trains (in single cases up to 200 trains), each having several coaches. Production (assembly) may happen in the assembly lines of the main contractor, but also at lines of suppliers. For instance, SIEMENS and Bombardier assemble trains of the same ordering process in their assembly lines in case of collaboration.

Depending on the train platform or modularization strategy, the degree of customization and configuration differs. In the investigated cases, selected trains are based on platform and module concepts. In the one case, it was applied to a regional train. The idea was to allow a dedicated flexibility of cabin design, but with controlled low inner variance. The instantiation of the design allowed outer variance constrained by configuration rules. Even options for train reconfiguration in operation (quick seat changes) and in longer cycles (door changes) were scoped within the design. In the other case, a high velocity train was designed with a platform concept integrating "black box modules" applied to zones.

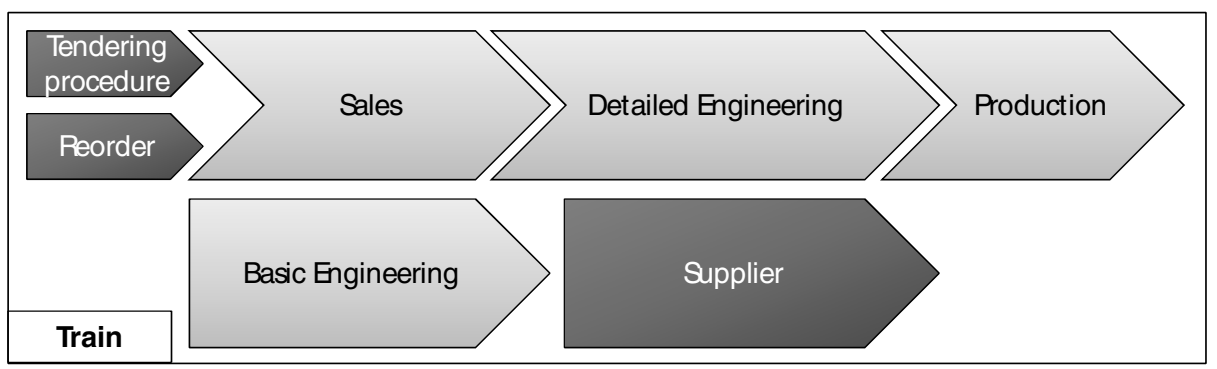

Fig. 4. Abstract representation of the train development process

The development process is comparable to the development process of plant engineering. An abstracted view is presented in figure 4. A basic design is developed independently to conceptualize a train and to implement new technology. The customization and instantiation is then happening in customer projects, mostly organized in tendering projects. The train manufacturer can participate in a tendering by specifying a train towards the customer requirements published in the tendering procedure. If a train manufacturer has questions about the requirements, his question will be answered and the question and answer are visible for all applicants. Customer contact after ordering the train is normal, but larger changes are not possible due to 
the initially defined requirements. If those requirements are changed, other applicants can later complain about the process.

After contracting, the detail design is performed based on the offered specification. Components and systems are developed with suppliers and accepted by the customer. Within the detail engineering, a common approach is to "copy" best-fit former projects and to use product structures and train components that fit best to the current project. Developed components can become available in new "standard catalogues" for later projects. The general product structure is more or less oriented at stakeholders but not used for customer-specific product configuration as trains are usually individually developed and sold in projects. Nevertheless, train manufacturers use to establish intern product configurators to raise the amount of reusability.

The configuration of seats, handles and buttons etc. is not constrained by fully defined configuration rules. In each development project, the cabin configuration is validated and designs are verified particularly. One reason is for instance security regulations and the particular use case (rail track, number of expected travelers, etc.). Another reason is the enormous count of variance caused by combination of existing design parameters like number of seats, kind of seat ("model"), required seat pitch, colors / materials, interfaces, and so on.

\section{Comparison of Investigated Industries and Mass Customization}

As seen in chapter 4 the need for mass customized highly complex products is given in the bus and train industry. Common for all the industries is the need for CSR even if the individual complexity is different. Mass Customization is mostly seen to offer methods for products with a finite solution space. Therefore the usage is limited for highly customizable products if it is not adjusted according to the industries' requirements.

Chapter 4 describes that bus industry and, increasingly, in train industry configuration tools for customer interaction are implemented. This is reasonable from a mass customization's point of view. Nevertheless, the authors' observation shows that today's configuration tools in these industries do not offer a complete coverage of the solution space. Even if the customers have different requirements for the configuration of trains and busses or even differing expectations for ordering travel coaches or city busses, a common configuration backend is needed. This configuration backend is required to supply either the customer or a sales person with the ability to create a suitable configuration including special requests with minimal costs.

The investigated industries do obviously use the concept of product modularity to gain benefits. Busses are divided into different zones within their layout which can more or less be configured separately. Trains incorporate black box modules with fully defined interfaces to enable configuration regarding power requirements for example. Variant management is hardly to divide from modularity. 
During the expert discussions the Fraunhofer IPK put together engineers from those different industries to discuss common problems and possible solutions for some or all industries. This expert discussion was focusing on product complexity. Its objective was to define product complexity, to find causes of product complexity, and to discuss ideas for management of product complexity.

Complexity from the point of view of the participant experts was seen to have an intensive relationship with number of product's elements, number of involved parties and breadth of project or product in general. To handle that complexity powerful models are necessary which are able to simply derive different views. The ATA chapters (Air Transport Association) used in the aircraft industry as a common documentation standard were seen to be an applicable way to ensure completeness and unambiguousness. Additionally, experts mentioned that there is a need for standard interfaces to bill of materials, PDM, ERP, MES as well as all the other domain-independent systems in the IT landscape with assured interoperability. In that context the questions that asks for the centralization of solutions is seen to be important. Those questions include how much every site should be able to establish their own local solutions. They also handle the question regarding the required steering mechanisms (production sites, customers, validation).

During a second expert discussion the Fraunhofer IPK focused on product customization. Therefore, causes and methods for managing product customization were discussed. The expert group was divided into two groups.

The first group discussed ideas for IT solution that provides "help" for customization. The discussed solution was called generic product structure. This solution was seen to be able to model project-specific " $100 \%$ product structures" by project-related reduction of elements of a " $150 \%$ product structure". The expected benefits were seen to provide the ability of trace linking to requirements management. This would allow evaluation of single solutions (functional, geometrical, and other aspects) and other beneficial functions.

The second group of experts discussed current and future challenges for product customization in general and solution approaches. Today's challenges were seen to be extended in future, namely customer-specific customization. Additionally, the extension of legal requirements and demographic change produce new requirements. Additionally legal requirements by the European Union and other institutions are seen to come up. Aim of the second group discussion was to gain knowledge about future challenges, together with a prioritization to address those challenges in the most useful order. Therefore the progress of these challenges have been evaluated and where supplemented with currently evolving and possible future challenges.

These expert discussions show that current and future requirements of highly individualized products are met by the aims of mass customization. Additionally, they show that methods and tools used within these industries need to be improved to apply mass customization to highly individualized, complex goods, and that improvements are on their way. 


\section{Conclusion}

This paper presents current methods and tools used for product development, sales, and production of mass-customized complex products with a high level of individualization. The results of an analysis of the bus and train industry yielded insights on typical industry requirements relevant for mass customization. It turned out that there is a need for open configurable areas in the product models since products like busses and trains must offer open solution spaces. Furthermore, a reduced use of configuration rules is required. Currently, the effort of developing and maintaining configuration rules exceeds the benefits of using configuration rules in some cases. This is why general mass customization methods are usually not implemented in the analyzed businesses in complete accordance with their description in literature. Instead, the analysis revealed that methods of mass customization are indeed implemented partially in the analyzed industries. Obviously, the benefits offered by mass customization are already recognized as potentials for highly customizable products. Solutions today are used in different levels.

Therefore, there exists a need for sophisticated structuring methods supported by intelligent tools to handle the complexity in the development of mass individualized products. Important is the above mentioned differentiation between configurable parts of a product and parts which are open for customer-specific requirements. Today's mass customization approaches do not cover parts of a product that are totally free to customize. An extended mass customizing approach which includes the option of open configuration is needed. Knowledge based engineering and model based systems engineering as well as other approaches seem to be promising in this context. An investigation of the applicability of such approaches should thus be done in following studies.

The presented approaches might also be valid and applicable to other branches and industrial products, such as plant engineering. In other industries there seems to be a stronger focus on technical aspects while esthetic aspects play a minor role. Such deviant demands of other industries should be analyzed in further studies. This paper delivers a starting point for the development of solutions for an extended mass customization approach mentioned before and the planning of further studies.

\section{References}

1. Kumar, A., Piller, F.T., Reismann, A., et al.: A Prioritization Algorithm for Configuration Scheduling in Mass Customization Environment. In: Mass Customization \& Personalization Conference (2007)

2. Moser, K.: Mass Customization Strategies. In: Mass Customization \& Personalization Conference (2007)

3. Wikström, S.: Value creation by company-consumer interaction. Journal of Marketing Management 12(5), 359-374 (1996)

4. Bettman, J.R., Johnson, E.J., Payne, J.W.: A Componentional Analysis of Cognitive Effort in Choice. In: Organizational Behavior and Human Decision Processes, vol. 45, pp. 111139 (1990) 
5. Petruzzellis, L., Somma, E.: Bundling, Mass Customization and Competition under Consumption Uncertainity. In: Mass Customization \& Personalization Conference (2007)

6. Chatterjee, S., Heath, T.B.: Conflict and Loss Aversion in Multiattribute Choice: The Effects of Trade-off Size and Reference Dependence on Decision Difficulty. In: Organizational Behavior and Human Decision Processes, vol. 67, pp. 144-155 (June 1996)

7. Mäkipää, M., Ahoniemi, L., Mertanen, M., et al.: The state of art of mass customization practices in Finnish technology industries - Preliminary results of multiple case study of 40 companies. In: Mass Customization \& Personalization Conference (2007)

8. Mertanen, M., Sievänen, M.: A Practical Approach to Mass Customization - Lessons Lerned from Finnish Machine Construction. In: Mass Customization \& Personalization Conference (2007)

9. Abramovici, M., Bellalouna, F., Neubach, M.: Delphi-Studie 2020 - Experteneinschätzung zur zukünftigen Entwicklung des Product Lifecycle Managements. Industrie Management (3) (2010)

10. Stark, R., Hayka, H., Israel, J.H., Kim, M., Müller, P., Völlinger, U.: Virtuelle Produktentstehung in der Automobilindustrie. Informatik Spektrum 34(1), 20-28 (2010)

11. Müller, P., Pasch, F., Drewinski, R., Hayka, H.: Kollaborative Produktentwicklung und digitale Werkzeuge, Defizite Heute - Potenziale Morgen. Fraunhofer IPK, CONTACT Software, VDI e.V., Berlin, Bremen, Düsseldorf (2013); Publisher: Stark, R., Drewinski, R., Hayka, H., Bredenbender, H.

12. Hahn, G.J., Doganaksoy, N., Hoerl, R.: The Evolution of Six Sigma. Quality Engineering 12(3), 317-326 (2000) 